\title{
CYCLE BIOLOGIQUE D'ACUARIA GRUVELI (GENDRE, 1913), NÉMATODE ACUARIDE PARASITE DU FRANCOLIN AU TOGO
}

\author{
J.-C. QUENTIN* et C. SEUREAU** \\ Collaboration technique : K. S. Agbalo* et A. Anglo**
}

RÉSUMÉ. Redescription d'Acuaria gruveli (Gendre, 1913), parasite, au Togo, de Francolinus bicalcaratus (Oiseau, Phasianidé). L'évolution des trois premiers stades larvaires obtenus par l'infestation expérimentale de l'Orthoptère Acrididae Tylotropidius patagiatus Karsch est décrite et figurée. La larve est infestante après 8 à 11 jours de développement à $30^{\circ} \mathrm{C}$. Cette larve s'apparente par sa morphologie caudale à celle d'A. hamulosa.

L'encapsulement des larves d' $A$. gruveli dans les muscles squelettiques de l'Insecte est comparé à celui des larves d'A. anthuris, d'A. depressa, et des Gongylonematidae. Cette comparaison met en évidence une meilleure adaptation à l'hôte intermédiaire chez les Acuarides que chez les Gongylonèmes.

\section{Life cycle of Acuaria gruveli (Gendre, 1913), an Acuarid Nematode parasite of the Double-spurred Francolin in Togo.}

SUMMARY. Redescription of Acuaria gruveli (Gendre, 1913), parasite of the phasianid bird Francolinus bicalcaratus in Togo. The development of the first three larval stages in experimentally infected Tylotropidius patagiatus Karsch (Orthoptera, Acrididae) is described and illustrated. Larvae are infective after 8 to 11 days of development at $30^{\circ} \mathrm{C}$. In caudal morphology, infective larvae are similar to those of $A$. hamulosa.

The encapsulation of $A$. gruveli larvae in the skeletal muscles of the insect is compared to the larval encapsulation of $A$. anthuris, $A$. depressa, and of the Gongylonematidae. In this respect, the Acuariidae appear better adapted than the Gongylonematidae to the intermediate host.

Les Francolins Francolinus bicalcaratus L. capturés dans la savane au Nord de Lomé, Togo, sont parasités dans une proportion de $85 \%$, au niveau de la muqueuse du gésier, par un Nématode Acuaride. Il s'agit de l'espèce Acuaria gruveli (Gendre, 1913) signalée à deux reprises (Gendre, 1912 et 1913) chez les Perdreaux du Dahomey.

Dans ce travail, nous précisons la morphologie adulte de cet Acuaride et réalisons expérimentalement son cycle évolutif afin de comparer son évolution larvaire à celle des Acuarides dont le cycle est connu.

\footnotetext{
* Laboratoire de Zoologie, École des Sciences, Université du Bénin, BP 1515 Lomé, Togo.

** Laboratoire d'Histophysiologie fondamentale et appliquée, ERA-CNRS 570, Université P. et M. Curie, 12 rue Cuvier, F 75005 Paris.

Accepté le I 5 mars I982.
} 


\section{Morphologie des adultes}

Corps extrêmement filiforme ( $f i g .1 \mathrm{~A}$ ) orné dans la région antérieure de 4 cordons céphaliques qui s'estompent en avant de la jonction œesophage-intestin chez le mâle, et au niveau de celle-ci chez la femelle. Structures céphaliques marquées par une dilatation cuticulaire médiane proéminente.

Mâle (fig. 1). Extrémité caudale vrillée sur elle-même, bourse caudale dissymétrique ( fig. $1 E, F)$ sans ornementation cuticulaire longitudinale, présentant trois à quatre paires de papilles préanales et six paires de papilles postanales dont cinq paires sont pédonculées. Il existe en outre une paire de phasmides à l'extrémité caudale. Spicules de tailles et de formes très inégales. Le spicule gauche est allongé avec une extrémité distale tronquée ( $f i g .1 H)$; le droit est court, très épais et creusé en gouttière.

Dimensions des mâles : longueur I2-I3 mm, largeur I 20 à $200 \%$, longueur des cordons céphaliques $800 \mu$, deirides, anneau nerveux et pore excréteur situés respectivement à I $70-2 I_{5} \mu, 220-$ $270 \mu$ et $320-380 \mu$ de l'apex, longueurs respectives du pharynx, de l'œesophage musculaire et de l'œsophage glandulaire $175^{-2}$ I $5 \mu, 940 \mu$ et $2050 \mu$; largeur de la bourse caudale $220 \mu$, dimensions du spicule droit $I_{70-1} 78 \mu \times 40 \mu$, dimensions du spicule gauche $550-670 \mu \times 17-14 \mu$; longueur de la queue $360-375 \mu$.

Femelle (fig. 2). Ovéjecteur représenté sur la fig. $2 E$.

Dimensions relevées sur une femelle longue de $29 \mathrm{~mm}$ : largeur $220 \mu$, longueur des cordons céphaliques I I 75-I $200 \mu$, deirides, anneau nerveux et pore excréteur situés respectivement à $190-220 \mu, 260$ et $360 \mu$ de l'apex ; longueurs respectives du pharynx, de l'œsophage musculaire et de l'œsophage glandulaire $200 \mu, \mathrm{I}$ I $50 \mu$ et I $800 \mu$; ovéjecteur situé à $13600 \mu$ de l'apex, dimensions des oufs $37 \times 25 \mu$, longueur de la queue $225 \mu$.

\section{Identification}

Les dimensions du corps, la longueur relative des cordons céphaliques, la morphologie et les dimensions des spicules permettent aisément d'identifier nos parasites à Acuaria gruveli (Gendre, 1913) récolté également chez Francolinus bicalcaratus au Dahomey.

\section{Cycle biologique}

Les œufs embryonnés des Acuarides sont placés entre les pièces buccales d'Orthoptères Acrididae appartenant à l'espèce Tylotropidius patagiatus maintenus en élevage à une température de $30^{\circ} \mathrm{C}$.

\section{1 - Allure du développement. Chronologie des mues.}

24 heures après l'infestation, des larves de premier stade ( fig. $3 A$ ) extrêmement actives, sont récoltées dans l'hémocoele de l'Insecte. Après trois jours, les larves n'apparaissent qu'en dilacérant les muscles reliant dorsalement les tergites abdominaux. Ces larves peu mobiles ont divisé leur cellule R1 et formé chez certaines d'entre 

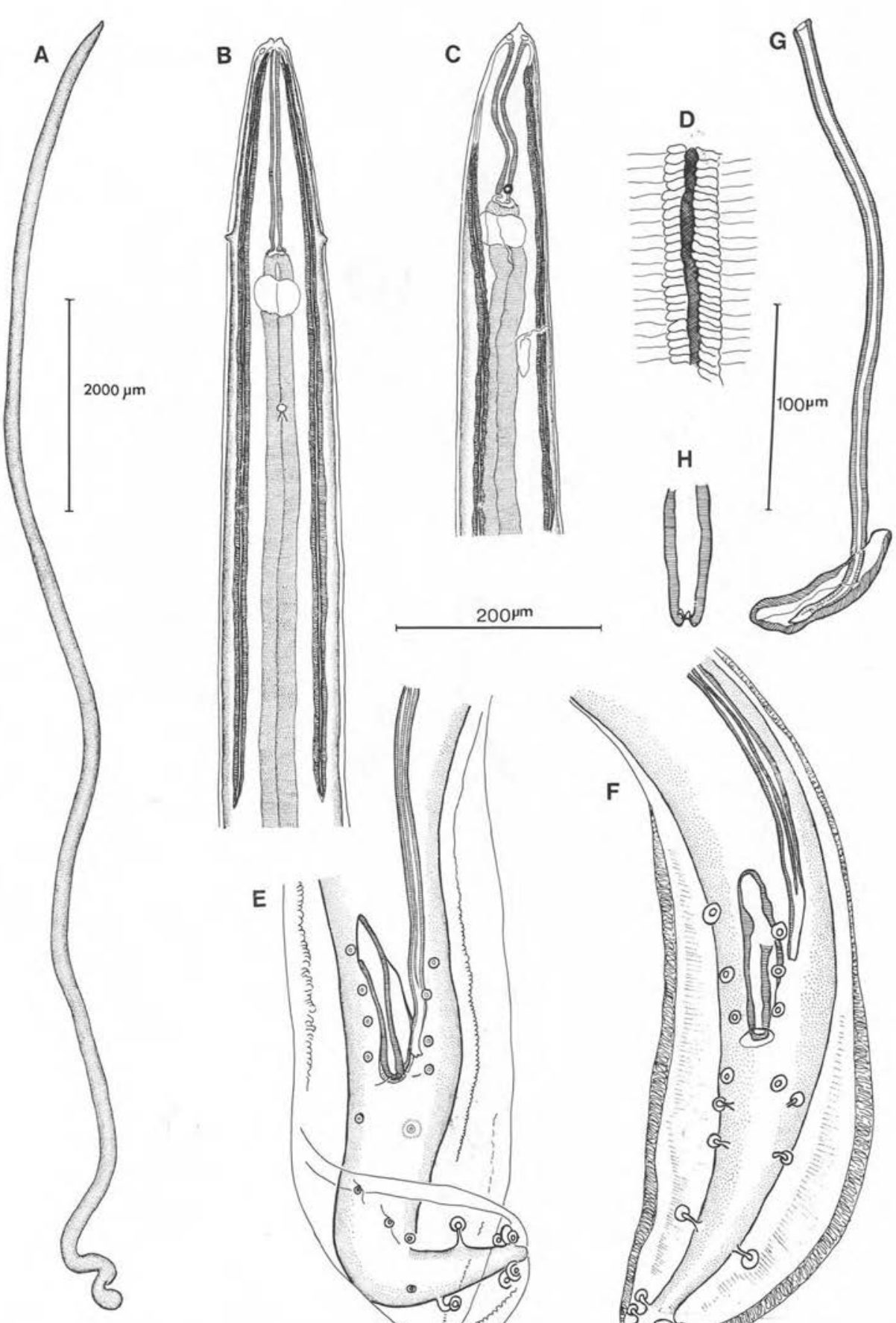

$20{ }^{\mu m}$

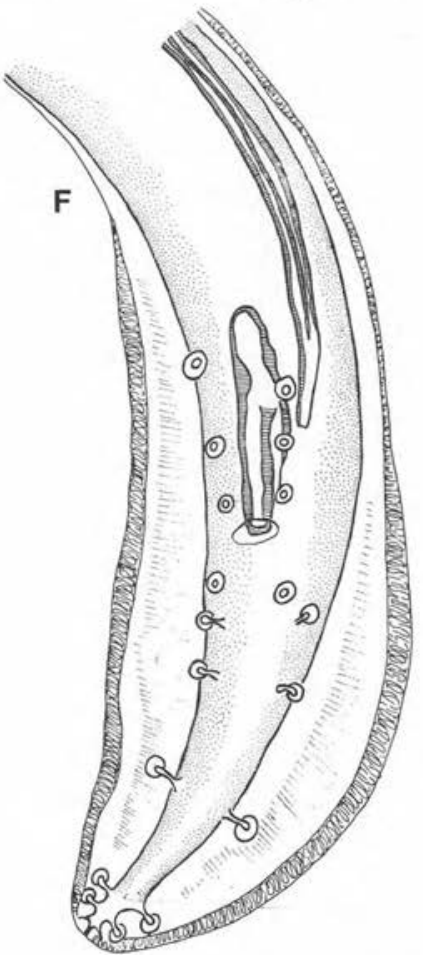

FIG. I. - Acuaria gruveli mâle. A : corps du mâle effilé sur toute sa longueur, enroulé en vrille au niveau de la bourse caudale ; B : extrémité antérieure du mâle, vue ventrale montrant le développement des cordons céphaliques; $\mathrm{C}$ : idem, vue latérale ; D : détail de l'ornementation cuticulaire au niveau des cordons céphaliques; $\mathrm{E}$ et $\mathrm{F}$ : bourses caudales de mâle, vue ventrale; $\mathrm{G}$ : spicules représentés en vue latérale ; $\mathrm{H}$ : détail de la pointe du spicule gauche.

A éch. $2000 \mu$, B, C, E, F et G éch. $200 \mu, \mathrm{D}$ et $\mathrm{H}$ éch. roo $\mu$. 

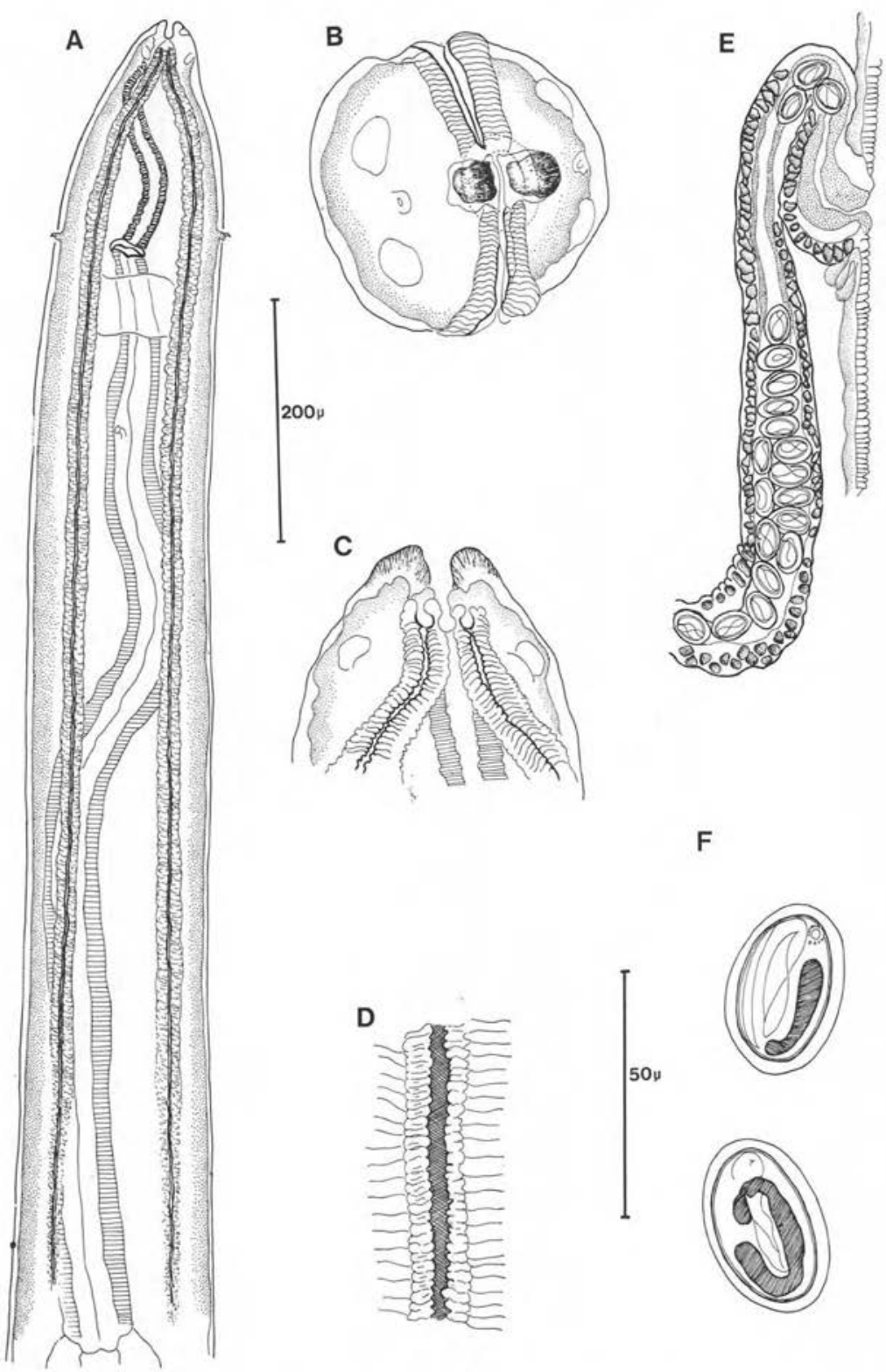

$\mathrm{F}$

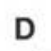

D

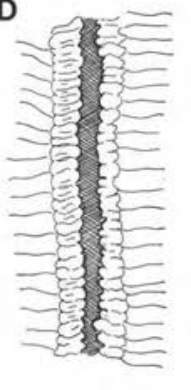

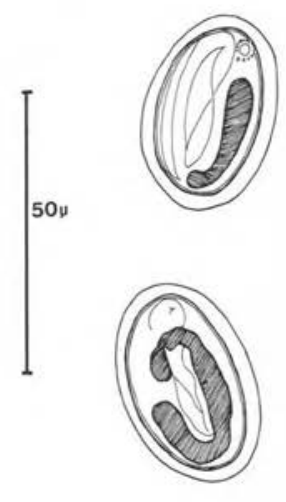

$50 y$

FIG. 2. - Acuaria gruveli femelle. A : extrémité antérieure du corps au niveau des cordons céphaliques, vue ventrale; $\mathrm{B}$ et $\mathrm{C}$ : tête représentée en vue apicale et en vue dorsale; $\mathrm{D}:$ détail de l'ornementation cuticulaire du cordon céphalique ; E : ovéjecteur; F : œufs avec corps interne riche en vitellus.

A et E éch. $200 \mu, \mathrm{B}, \mathrm{C}, \mathrm{D}$ et $\mathrm{F}$ éch. $50 \mu$. 

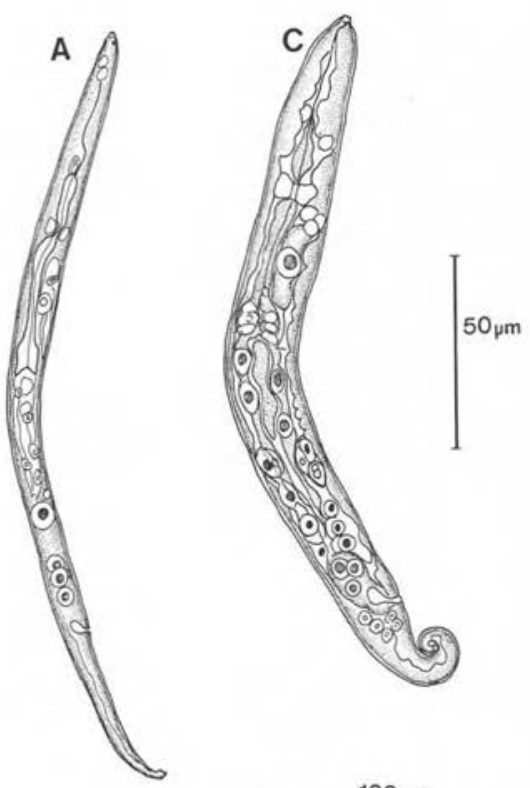

E
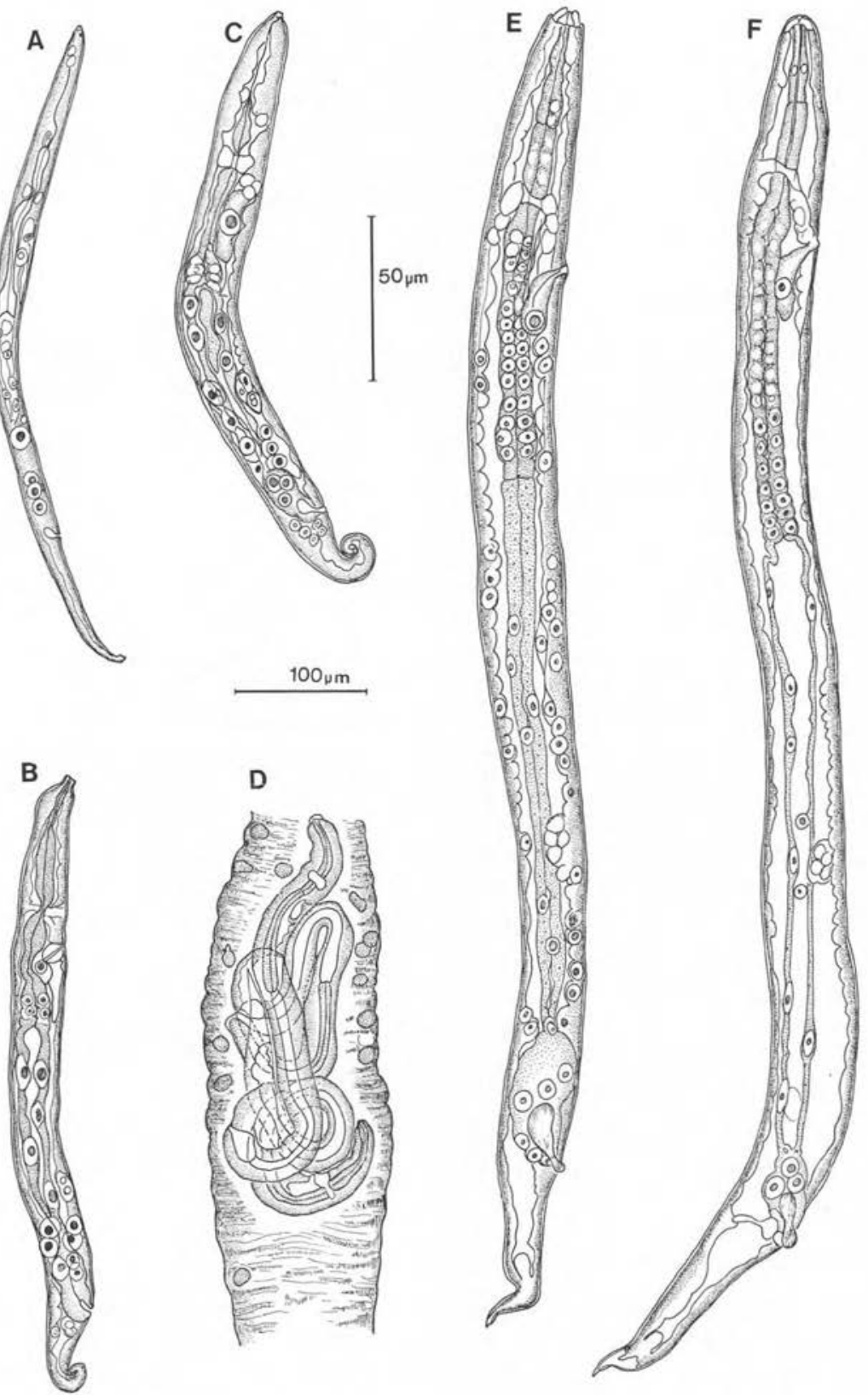

FIG. 3. - Acuaria gruveli premier stade larvaire. A : larve de 24 heures après l'infestation ; $\mathrm{B}$ et $\mathrm{C}$ : larves 3 jours après l'infestation; $\mathrm{D}:$ larves en fin de premier stade à l'intérieur d'une fibre musculaire; $\mathrm{E}$ et $\mathrm{F}$ : détail de ces larves à la fin du premier stade.

A, B, C, E et F éch. $50 \mu$, D éch. $100 \mu$. 
elles les cordons mésodermiques ( $f$ g. $3 B$ et $C$ ). La pénétration à l'intérieur des fibres musculaires est donc rapide, et à 5 jours, vers la fin du premier stade, plusieurs larves peuvent être observées à l'intérieur de la même fibre (fig. $3 D$ ). A six jours, les larves sont au deuxième stade larvaire, et au $7 \mathrm{e}$ jour, la courbure dorsale de la région postérieure, caractéristique du stade infestant, affecte déjà la partie caudale. La deuxième mue survient au $8^{\mathrm{e}}$ jour du développement.

\section{2 - Croissance larvaire.}

Elle est marquée par la faible croissance au cours du deuxième stade. La larve grandit au premier stade de 200 à $400 \mu$, au deuxième stade de 420 à $520 \mu$. Elle mesure 550 à $610 \mu$ au troisième stade.

\section{Dimensions des larves et de leurs organes.}

Premier stade larvaire :

- larve âgée de 24 heures (fig. $3 A$ ). Longueur $200 \mu$, largeur au niveau du pore excréteur $9 \mu$; anneau nerveux et pore excréteur situés à 53 et $66 \mu$ de l'apex, longueurs de l'oesophage et dé l'intestin 90 et $35 \mu$, cellule RI distante de l'anus de $32 \mu$, longueur des 3 cellules rectales $22 \mu$, longueur de la queue $45 \mu$.

— larve âgée de 3 jours avec début de division des cellules RI ( $\mathrm{ig}$. $3 B$ ). Longueur $22 \mu$, largeur I6 $\mu$, anneau nerveux et pore excréteur situés à $4 \mathrm{I}$ et $5 \mathrm{I} \mu$ de l'apex, longueurs de l'œesophage et de l'intestin 77 et $70 \mu$; cellules issues de RI distantes de l'anus de $26 \mu$, longueur des cellules rectales $22 \mu$, distance de l'ébauche génitale à l'anus $3^{8} \mu$, longueur de la queue $40 \mu$.

- larve âgée de trois jours avec formation des cordons mésodermiques ( fig. $3 C$ ). Longueur 2 ro $\mu$, largeur $19 \mu$, anneau nerveux et pore excréteur situés à 47 et $5^{8} \mu$ de l'apex; longueurs de l'œsophage $83 \mu$ et de l'intestin $62 \mu$, longueur des cellules rectales $2 \mathrm{I} \mu$, distance de l'ébauche génitale à l'anus $40 \mu$, longueur de la queue $40 \mu$.

- larve âgée de 5 jours avec décollement de l'exuvie dans les régions céphaliques et caudales (fig. $3 E$ et F). Longueur $45^{\circ} \mu$, largeur $24 \mu$, anneau nerveux et pore excréteur situés à 52 et $72 \mu$ de l'apex, longueurs respectives de l'ébauche pharyngienne, de l'œesophage et de l'intestin $25 \mu$, I6o $\mu$, et I $85 \mu$ longueur du rectum $29 \mu$, distance de l'ébauche génitale à l'anus I9o $\mu$, longueur de la queue $7^{2} \mu$.

Deuxième stade larvaire, marqué par un allongement brutal de la région caudale et par la différenciation du tube pharyngien :

- larve âgée de 6 jours avec pharynx tubulaire différencié (fig. $4 B$ ). Longueur $420 \mu$, largeur $26 \mu$, anneau nerveux et pore excréteur situés à 57 et $72 \mu$ de l'apex, longueurs respectives du pharynx, de l'œsophage et de l'intestin $30 \mu$, I $23 \mu$ et $175 \mu$, longueur du rectum $28 \mu$, distance de l'ébauche génitale à l'anus $100 \mu$, longueur de la queue $67 \mu$.

- larve âgée de 7 jours (fig. ${ }_{4}$ C). Longueur $520 \mu$, largeur $33 \mu$, anneau nerveux et pore excréteur situés à 63 et $72 \mu$ de l'apex, longueurs respectives du pharynx, de l'œsophage musculaire et de l'œsophage glandulaire 60,155 et $145 \mu$, longueur du rectum $29 \mu$, distance de l'ébauche génitale à l'anus $125 \mu$, longueur de la queue $80 \mu$.

\section{3 - Morphologie du troisième stade larvaire (fig. 5).}

La larve infestante d'Acuaria gruveli est, au repos, courbée sur la face ventrale. En arrière de l'ébauche génitale, l'extrémité caudale se rabat brusquement sur la face dorsale et effectue une boucle sur elle-même ( $f i g .5 \mathrm{~A}$ ). Cette larve est caractérisée par un tassement de la région intestinale, elle présente deux ailes latérales. Les structures céphaliques en vue apicale sont représentées sur la figure 5C. L'ornementation caudale ( fig. $5 F$ ) est composée d'une pointe médiane épaisse bordée latéralement par deux pointes plus petites et plus fines, et dorsalement par une courte digitation. 


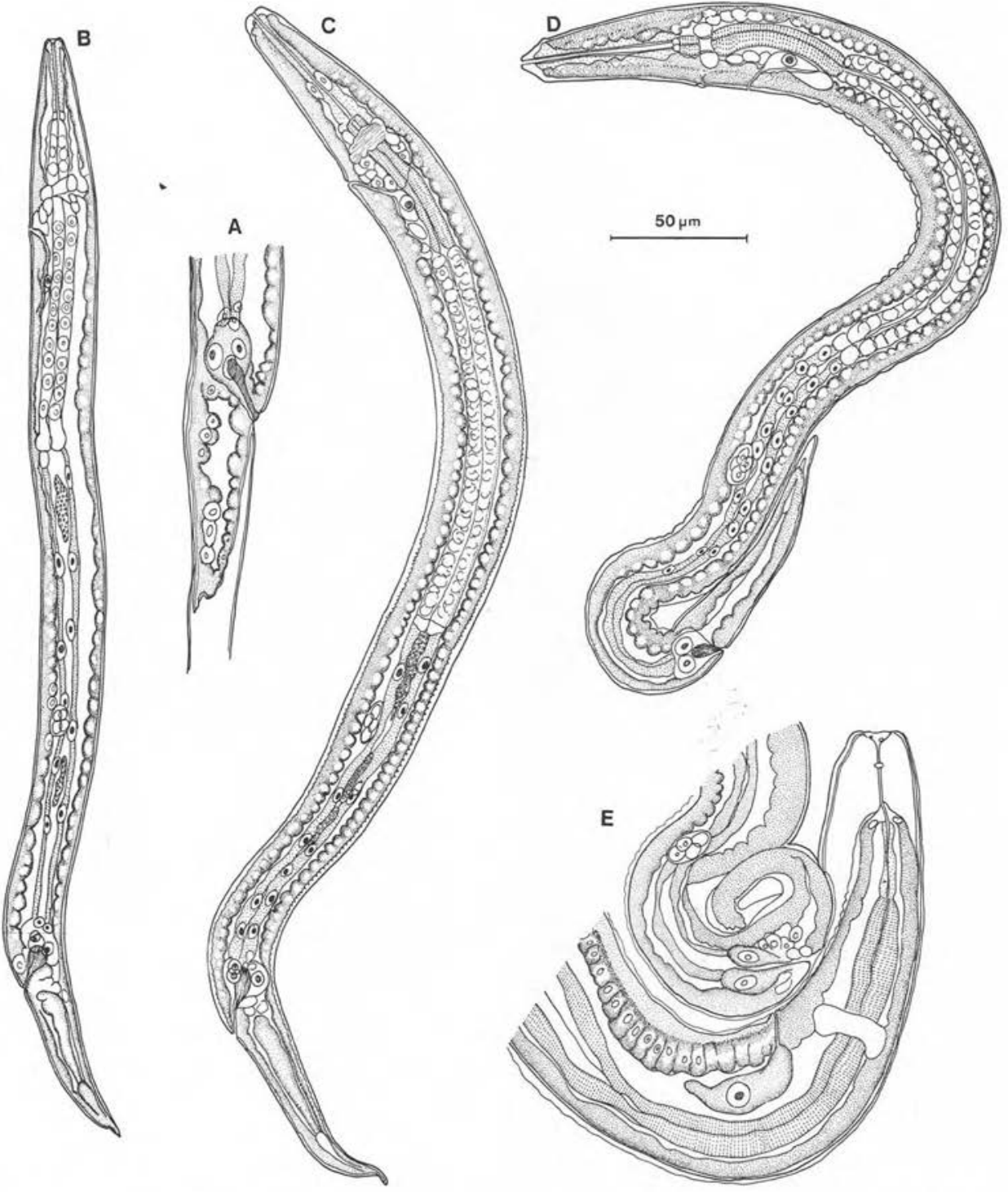

FIg. 4. - Acuaria gruveli deuxième stade larvaire. A : $\mathrm{I}^{\mathrm{re}}$ mue, queue de larve de deuxième stade dans l'exuvie du premier stade ; B : larve âgée de 6 jours ; C : larve âgée de 7 jours; D : larve âgée de 8 jours, fin du deuxième stade, montrant la courbure dorsale caractéristique de la région caudale des larves infestantes d'Acuaria; E : détail des régions céphaliques et caudales d'une larve âgée de II jours en train de muer.

A, B, C, D et E éch. $50 \mu$. 

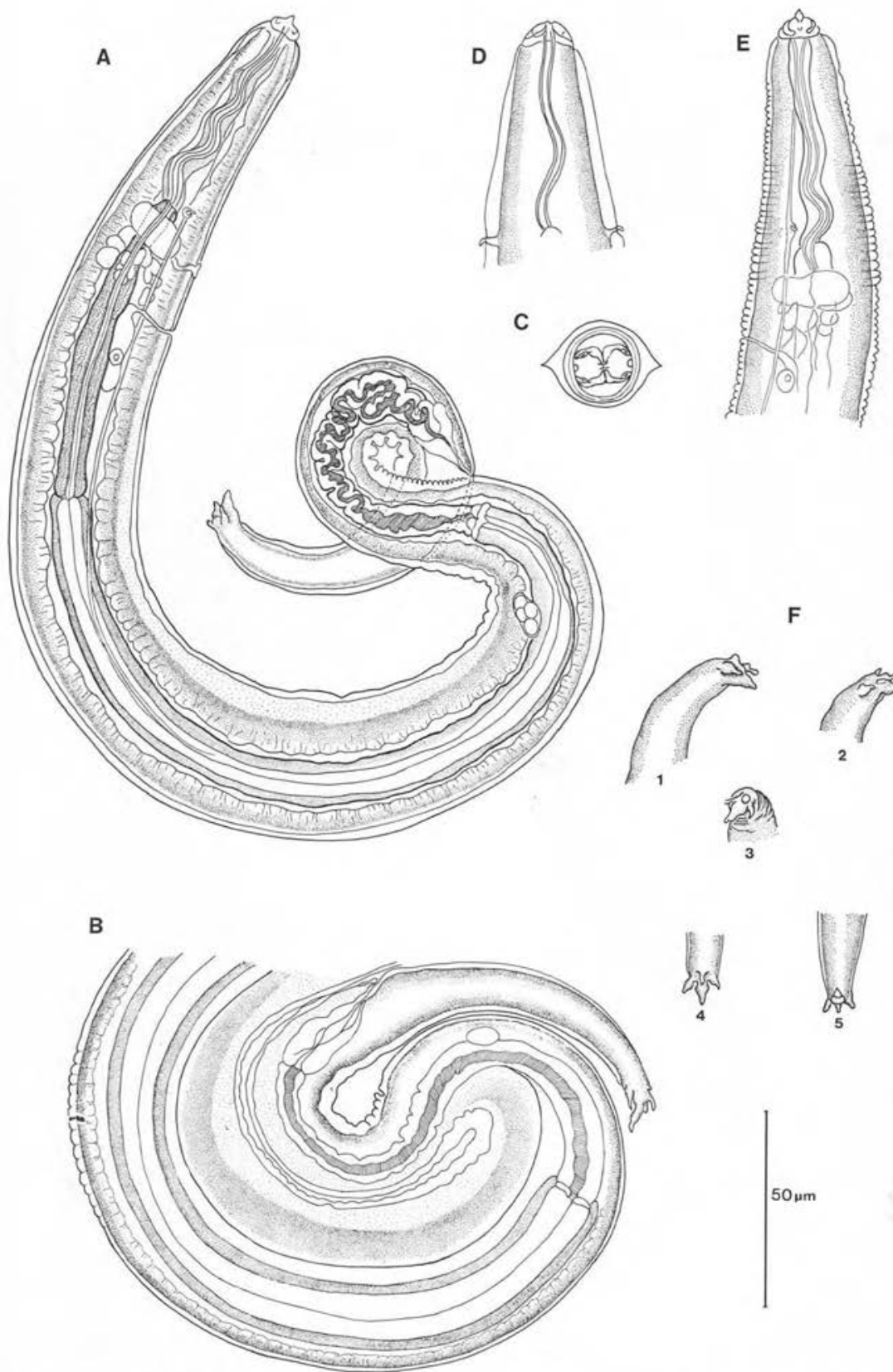

FIG. 5. - Acuaria gruveli troisième stade larvaire. A : larve en vue latérale; $\mathrm{B}$ : détail de la courbure dorsale de la région caudale ; C : tête en vue apicale; D et E : extrémité antérieure, région pharyngienne représentée en vue ventrale et latérale ; F : détail de l'ornementation de l'extrémité caudale représentée successivement : en vue latérale (I), en vue oblique (2), en vue apicale (3), en vue ventrale (4) et en vue dorsale (5).

A, B, C, D, E et F éch. $50 \mu$. 


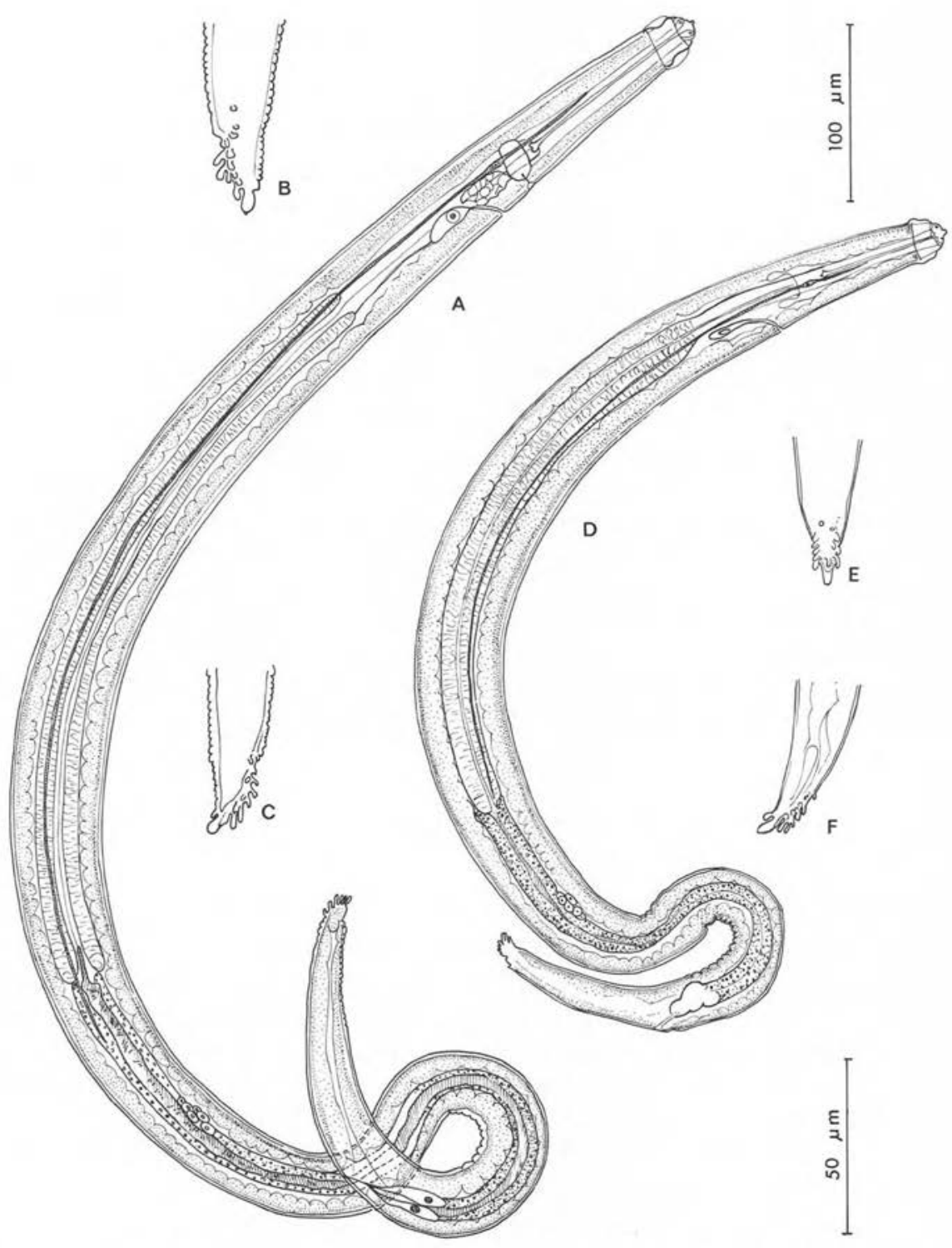

Fig. 6. - Acuaria depressa (Schneider, I866) et $A$. anthuris (Rud., I8I9). A : larve d'A. depressa en vue latérale; $\mathrm{B}$ et $\mathrm{C}$ : détail de l'ornementation caudale; $\mathrm{D}:$ larve $\mathrm{d}$ ' $A$. anthuris en vue latérale; $\mathrm{E}$ et $\mathrm{F}$ : détail de l'ornementation caudale.

A et D éch. Ioo $\mu, \mathrm{B}, \mathrm{C}, \mathrm{E}$ et F éch. $50 \mu$. 
Dimensions des larves infestantes : longueur 540-610 $\mu$, largeur 30 à $44 \mu$; deirides, anneau nerveux et pore excréteur situés respectivement à $54-57 \mu, 66-67 \mu$ et $82-88 \mu$ de l'apex; longueurs respectives du pharynx, de l'œsophage musculaire et de l'œsophage glandulaire $60 \mu, 78-85 \mu$ et $230-240 \mu$, longueur de l'intestin $\mathrm{IO}_{5} \mathrm{I} 67 \mu$; ébauche génitale située à $22 \mu$ de la pointe caudale, longueur de la queue $90-92 \mu$.

\section{4 - Encapsulement cellulaire des larves infestantes chez l'Insecte.}

Localisation. 15 jours après l'infestation expérimentale, l'examen histologique de plusieurs Criquets parasités permet de constater que toutes les larves infestantes d'Acuaria gruveli sont localisées dans des fibres musculaires. La plupart de ces larves parasitent les muscles squelettiques intersegmentaires de l'abdomen, mais on peut également en observer quelques-unes dans la musculature thoracique. Chaque fibre parasitée héberge en général une seule larve, plus rarement deux ou plus ( fig. $3 \mathrm{D}$ et $7 \mathrm{~A}$ ). Dans le cas où une fibre ne contient qu'une seule larve, celle-ci est disposée longitudinalement par rapport à la fibre, ou, plus souvent, enroulée sur elle-même.

Histopathologie de la fibre parasitée. Les larves infestantes occupent une cavité creusée à l'intérieur des fibres musculaires. Cette disposition entraîne une dilatation locale de la fibre, sans qu'il y ait toutefois rupture du sarcolemne et de la fine enveloppe conjonctive qui l'entoure ( $f \mathrm{~g} .7 \mathrm{~B}$ ). Sauf pour quelques noyaux ( $\mathrm{fig} .7 \mathrm{~A}$ ), les fibres parasitées ne présentent pas de signes d'hypertrophie ; les faisceaux de myofibrilles sont souvent désorganisés. On note également une lyse partielle de ces myofibrilles au pourtour de la cavité contenant les parasites (fig. $7 \mathrm{C}$ ). Aucune accumulation anormale d'hémocytes n'a été observée tant à l'intérieur qu'à l'extérieur des fibres parasitées. Il n'apparaît, de même, aucun dépôt pigmentaire.

\section{Discussion}

Les formes larvaires infestantes d'Acuaria gruveli peuvent être comparées à celles de trois autres espèces d'Acuaria dont les cycles sont connus.

- A. depressa (Schneider, 1866). Cram (1934) en a réalisé le cycle à partir d'Acuaria parasites de Corvus brachyrynchos. Nous avons également obtenu la larve infestante à partir d' $A$. depressa récolté chez la Corneille Corvus corone, en infestant expérimentalement des Criquets Locusta migratoria (fig. $6 A, B, C$ ). Cette larve est de grande taille (1,2 à $1,4 \mathrm{~mm}$ de long) et présente une ornementation caudale constituée de deux rangées ventrales de sept à huit digitations, et d'une pointe caudale plus trapue surmontée d'une digitation dorsale.

- A. anthuris (Rud., 1819). Son cycle biologique expérimental a été réalisé à partir d'Acuaria récoltés chez la Pie Pica pica (Quentin et coll., 1972). La larve infestante obtenue chez Locusta migratoria (fig. $6 D, E, F$ ) présente une ornementation caudale comparable à celle d' $A$. depress $a$ avec toutefois des digitations latérales moins nombreuses. Cependant la taille du troisième stade larvaire d'A. anthuris est plus petite (860 $\mu$ de long, sur $50 \mu$ de large) que celle du troisième stade d'A. depressa. 

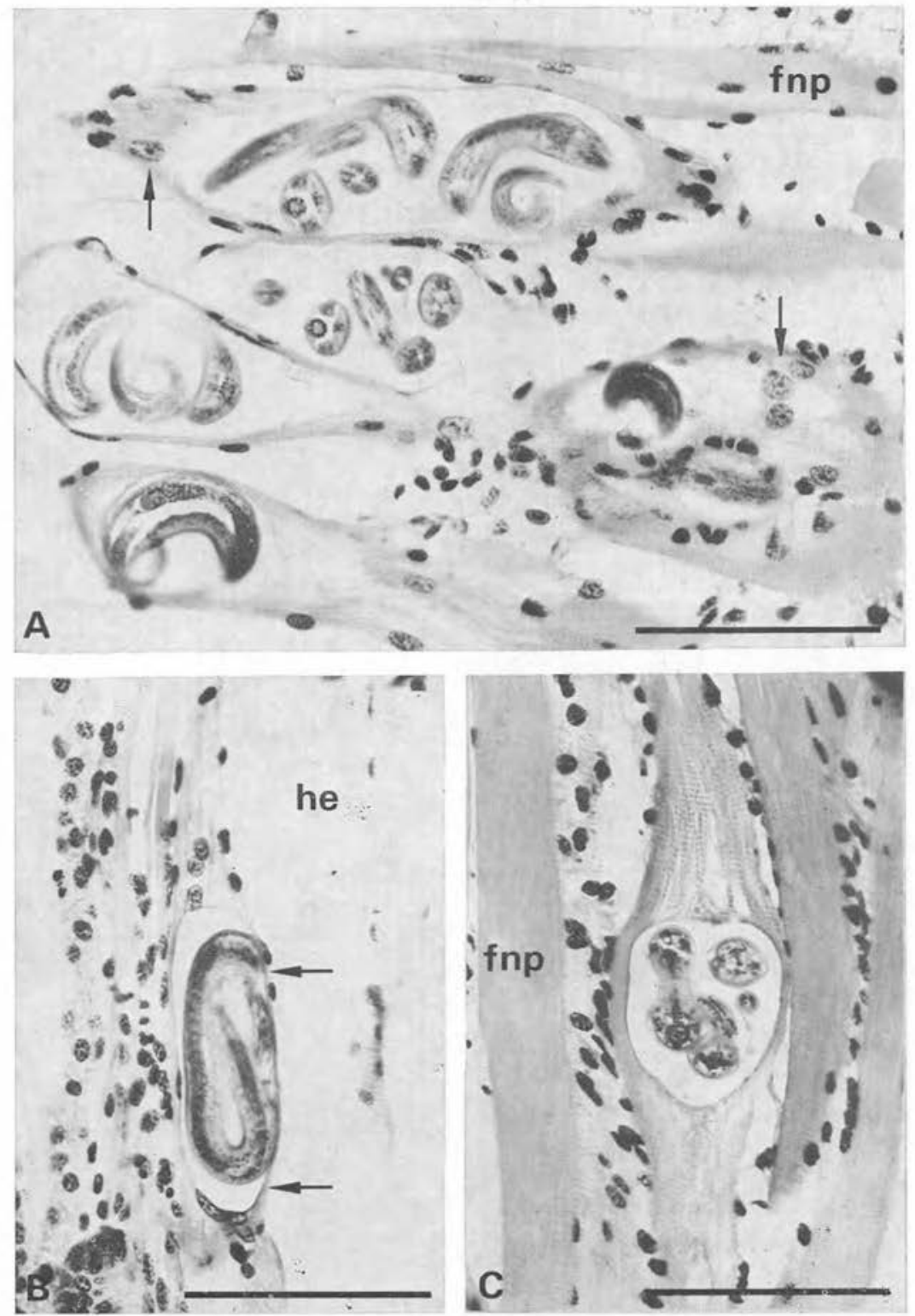

FIG. 7. - Encapsulement des larves d'Acuaria gruveli chez l'Insecte. A : 8 jours après l'infestation expérimentale, nombreuses larves dans les fibres d'un faisceau musculaire. Quelques noyaux de fibres parasités sont hypertrophiés (flèches). B : 8 jours après l'infestation, une larve n'est séparée de l'hémocoele(he) que par une fine structure provenant de la membrane et de l'enveloppe conjonctive de la fibre parasitée (flèches). C : I4 jours après l'infestation, la fibre parasitée présente une dissociation des myofibrilles.

fnp : fibre musculaire non parasitée, éch. roo $\mu$. 

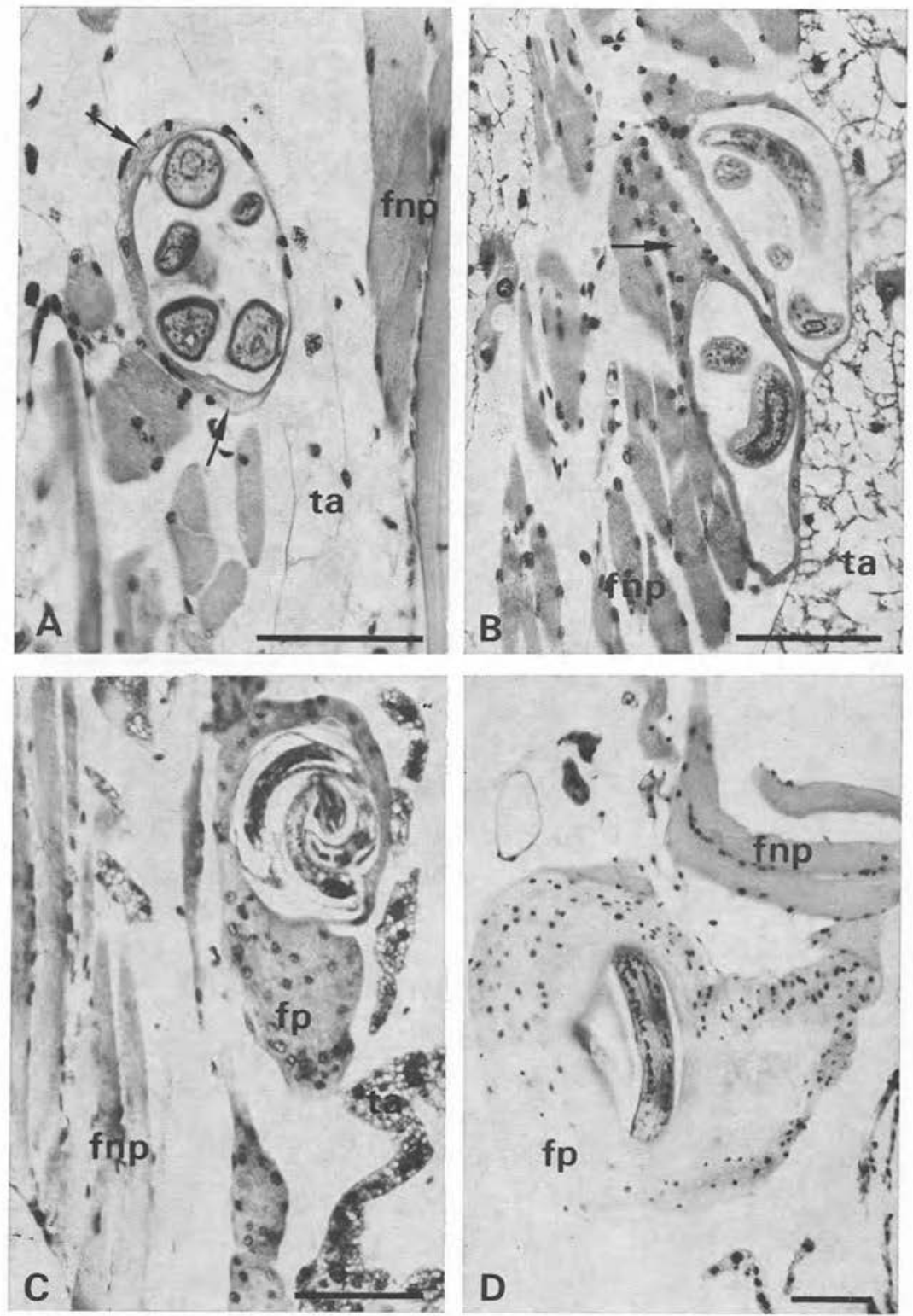

FIG. 8. - Encapsulement des larves d'Acuarides et de Gongylonèmes chez l'Insecte. A : A vuaria anthuris, 15 jours après l'infestation expérimentale. $\mathrm{B}: A$. depressa, 20 jours après l'infestation. Dans ces deux cas, la fibre musculaire ne réagit que par une légère lyse myofibrillaire (flèches) à la présence des parasites. C : Gongylonema brevispiculum, 30 jours après l'infestation. D : Gongylonema mucronatum, 56 jours après l'infestation. Dans ces deux autres cas, la fibre parasitée (fp) réagit par une hypertrophie du sarcoplasme sans hypertrophie des noyaux musculaires.

fnp : fibre musculaire non parasitée, ta : tissu adipeux, éch. Ioo $\mu$. 
La différence constante de taille des larves infestantes confirme la séparation des deux espèces $A$. depressa et $A$. anthuris proposée par Chabaud et Petter (1961) d'après l'étude morphologique des adultes. De ce fait, nous pensons que les larves infestantes d'Acuaria obtenues par Rietschel (1973) à partir d'adultes recueillis chez Corvus corone en Allemagne doivent être identifiées à $A$. depressa et non à $A$. anthuris.

La larve infestante d'A. gruveli diffère de celles d'A. depressa et d'A. anthuris par sa petite taille $(540-610 \mu)$ et par son ornementation caudale munie de quatre pointes.

- A. hamulosa (Diesing, 1851). Alicata (1937) en a réalisé le cycle à partir d'adultes parasites de poulets et de dindes. La larve infestante se rapproche morphologiquement de $A$. gruveli par son ornementation caudale et la flexure très prononcée de sa région postérieure. Elle témoigne, malgré une taille plus importante $(735-750 \mu)$, d'une étroite parenté entre les deux espèces $A$. gruveli et $A$. hamulosa.

En ce qui concerne l'encapsulement des larves infestantes chez l'Insecte-hôte intermédiaire, la localisation et le type de capsule d'Acuaria gruveli sont en tous points comparables à ce que nous avons observé avec Acuaria anthuris (Quentin et coll., 1972) (fig. 8A) et Acuaria depressa (fig. 8B). Pour ces trois Acuarides du genre Acuaria, les larves provoquent seulement une désorganisation de la fibre parasitée due à la taille relativement importante de ces Nématodes par rapport au diamètre moyen des fibres musculaires. Dans tous les cas, on ne note aucune manifestation d'hypertrophie cellulaire habituellement observée au cours de l'encapsulement de la plupart des Nématodes Spirurides dans d'autres tissus de l'Insecte (Seureau, 1973).

Un autre groupe de Nématodes Spirurides, les Gongylonèmes, parasite également les fibres musculaires de Criquets après une infestation expérimentale. Toutefois, et contrairement à ce que nous avancions pour Gongylonema brevispisculum (Quentin et Seureau, 1978), les larves infestantes de ce Gongylonème ( $f i g .8 C$ ) comme celles de Gongylonema mucronatum (fig. $8 D$ ) provoquent une nette réaction d'hypertrophie de la fibre musculaire parasitée. La paroi de la capsule musculaire peut atteindre 150 à $200 \mu$ d'épaisseur, ce qui n'est jamais le cas avec les Acuarides. L'hypertrophie de la fibre ne concerne que le sarcoplasme ; les noyaux ayant la même taille et la même structure que les noyaux des fibres non parasitées.

Ainsi, les larves infestantes des Gongylonèmes et celles des Acuarides ont un encapsulement différent, bien que parasitant le même tissu de l'Insecte, le tissu musculaire.

Comparés aux Gongylonèmes, les Acuarides montrent une absence de réaction cellulaire du tissu parasité que l'on peut interpréter comme l'expression d'un plus haut degré d'adaptation à l'hôte intermédiaire.

Remerciements : Nous remercions vivement le Professeur Johnson, Recteur de l'Université du Bénin, pour les facilités qu'il a bien voulu nous accorder dans la réalisation de ce travail. 


\section{BIBLIOGRAPHIE}

Alicata J. E. : The gizzard worm Cheilospirura hamulosa and its transmission to chickens in Hawaii. U. S. Dept. Agric. Exp. Sta. Arc., 1973, 2, I-7.

Chabaud A. G., Petrer A. : Nématodes du genre Acuaria de la Faune de France. Ann. Parasitol. Hum. Comp., 1961, 36, 409-424.

CRAM E. B. : Recent records of the gizzard worm Acuaria anthuris (Rudolphi, I819) (Nématode : Acuariidae) with observations on its life history. Proc. helminth. Soc. Wash., I934, $r, 49-50$.

Gendre E. : Sur quelques espèces de Dispharages du Dahomey. Proc.-verb. Soc. Lin. Bordeaux, $1912,66,31-39$.

GENDRE E. : Sur une espèce nouvelle de Dispharage. Proc.verb. Soc. Lin. Bordeaux, 1913, 67, $87-88$.

Quentin J. C., Seureau C., Gabrion C. : Cycle biologique d'Acuaria anthuris (Rudolphi, I8I9) Nématode parasite de la Pie. Z. Parasitenk., 1972, 39, 103-126.

Quentin J. C., Seureau C. : Identification et biologie du Gongylonème parasite du Macroscélide en Tunisie. Ann. Parasitol. Hum. Comp., 1978, 53, 631-640.

RIETSCHEL G. : Untersuchungen zur Entwicklung einiger in Krähen (Corvidae) vorkommender Nematoden. Z. Parasitenk., 1973, 42, 243-250.

Seureau C. : Réactions cellulaires provoquées par les Nématodes Subulures et Spirurides chez Locusta migratoria (Orthoptère) : localisation et structure des capsules. Z. Parasitenk., 1973, $4 I$, II $9-138$. 